\title{
Substituting EMC emission measurement by field and cable scan method using measured transfer function
}

\author{
D. Rinas, J. Jia, A. Zeichner, and S. Frei \\ Dortmund University of Technology, Dortmund, Germany \\ Correspondence to: D. Rinas (denis.rinas@tu-dortmund.de)
}

\begin{abstract}
Today EMC emissions of automotive components are often measured in anechoic chambers by an antenna at fixed position according to CISPR 25 (ALSE-method). The antenna voltage often cannot sufficiently describe the behaviour of the measured electronic components and systems. Furthermore space requirements and costs are very high for the ALSE-method. Field- and cable-scan methods combined with near-field to far-field transformation techniques might be a good alternative.

Residual reflections from the walls, the metallic floor, the measuring table, interaction of the antenna with the environment, and other factors affect the measurements. Thus, models which only regard the current distribution for near- and far field calculation cannot produce results equal to a chamber measurement.

In this paper methods for computing transfer functions for the substitution of EMC antenna measurements with fieldand cable scans in a specified calibration area are introduced. To consider influences of the environment, the environment is characterized in a first step and included with transfer functions in the calculation process for the equivalent ALSEfield.
\end{abstract}

\section{Introduction}

Standardized component field measurement methods, like the ALSE antenna method defined e.g. in CISPR 25 (2007) for evaluation of electro-magnetic emissions from automotive systems, suffer from the need of large and expensive anechoic chambers. Also a single field strength value is often not sufficient to characterize the EMI behavior of a complex system. Residual reflections from the walls, the metallic floor, the measuring table, interaction of the antenna with the environment, and other influences affect the measurements and reduce reproducibility. Furthermore it is not possible to use

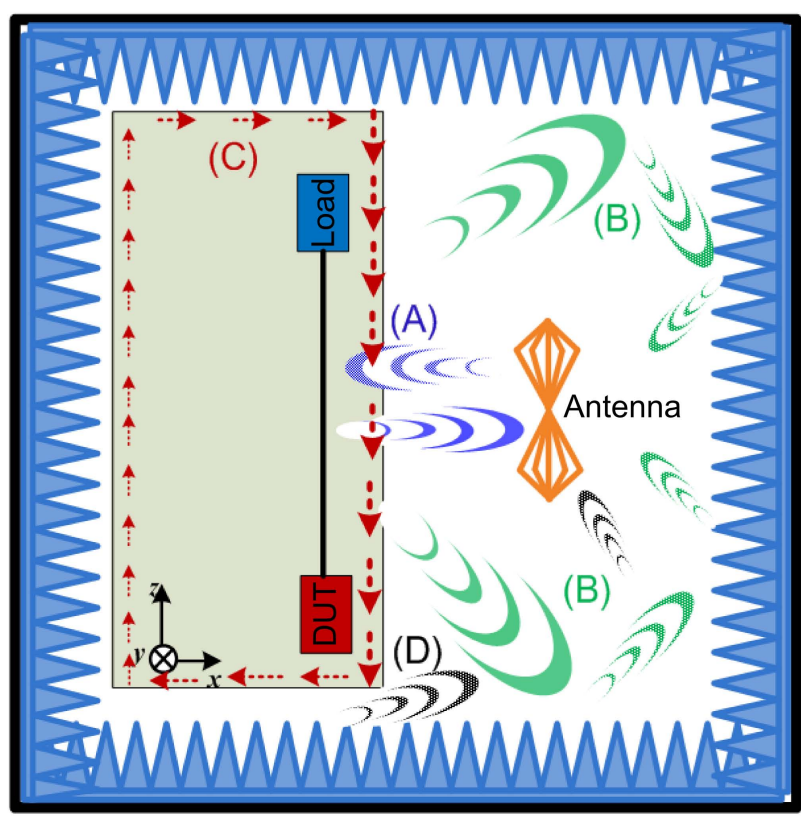

Fig. 1. Influencing factors of CISPR 25 ALSE method.

the measurement data for behavioral simulation model creation. Alternative methods are necessary.

Basically the electromagnetic emission can be distinguished in the emission of printed circuit boards with housing and the emission of the connecting cable bundles. The behavior of the cable can be obtained by cable scan methods where the dominant common-mode current is used to create an emission model (Jia et al., 2012). Time domain measurements give the possibility to get amplitude and phase information. Alternatively phase retrieval algorithm based on current distribution measurements could identify in some cases the phase based on amplitude-only frequency domain measurements. To compute an emission model of PCBs 


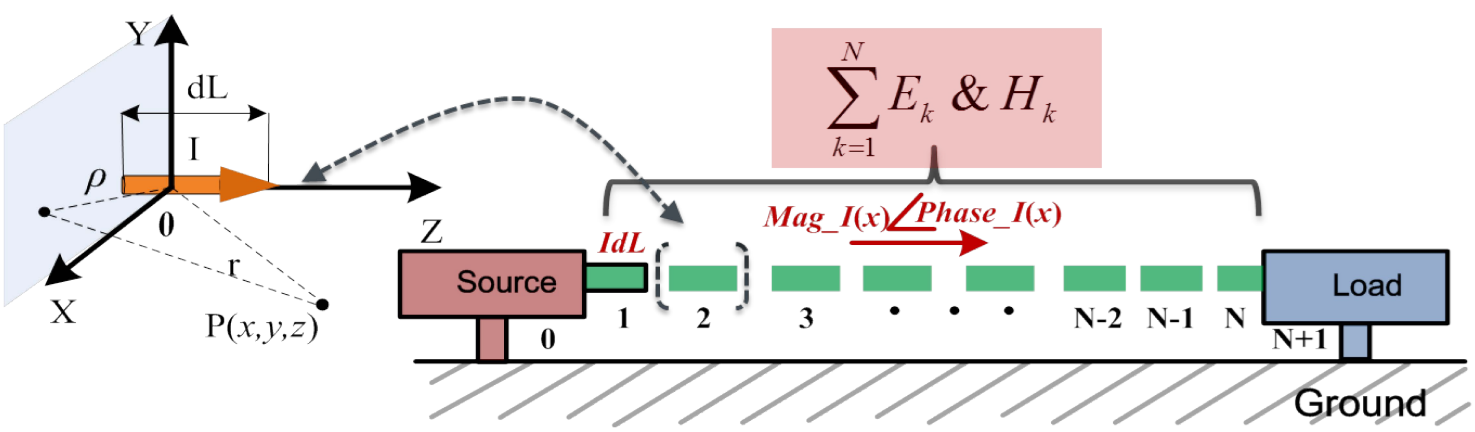

Fig. 2. Multiple-dipole-model for a cable bundle.
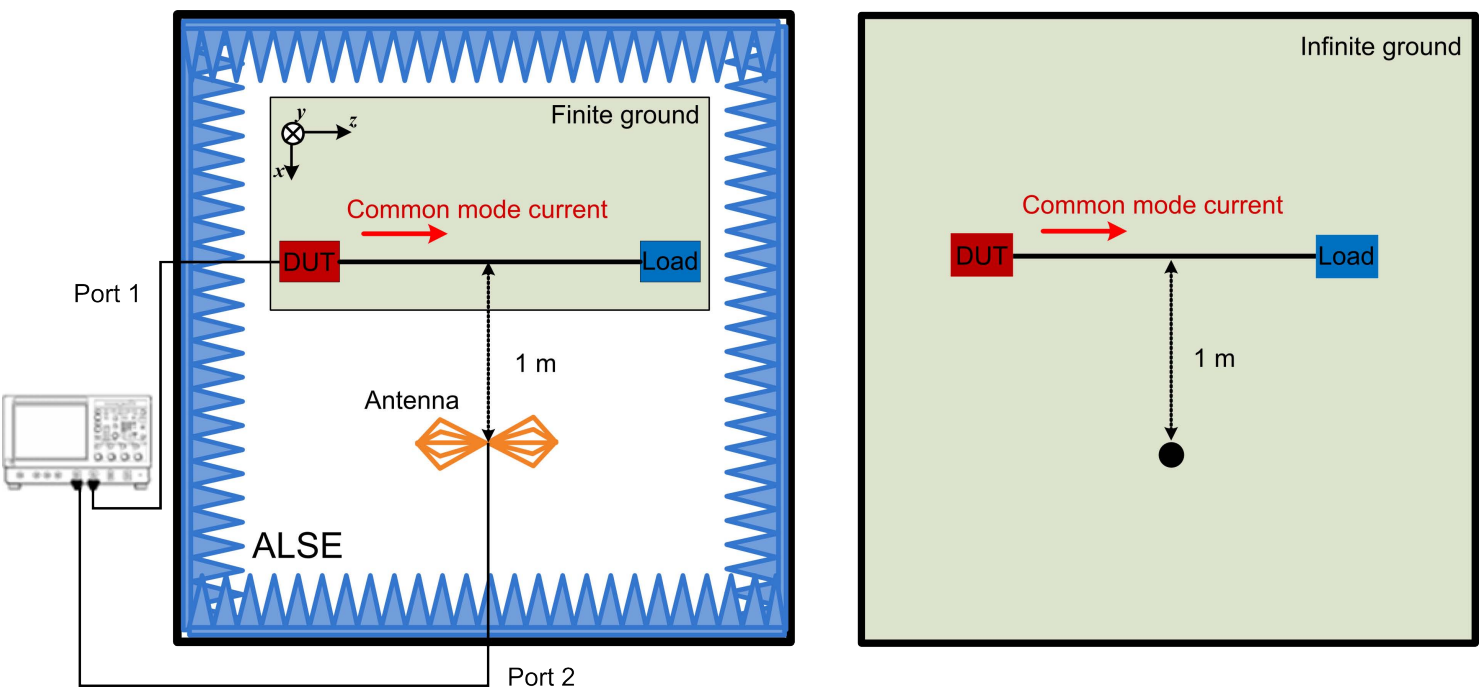

Fig. 3. CISPR 25 setup and simulation model. (a) Antenna measurement from cable bundle. (b) Field simulation from cable bundle by MoM.

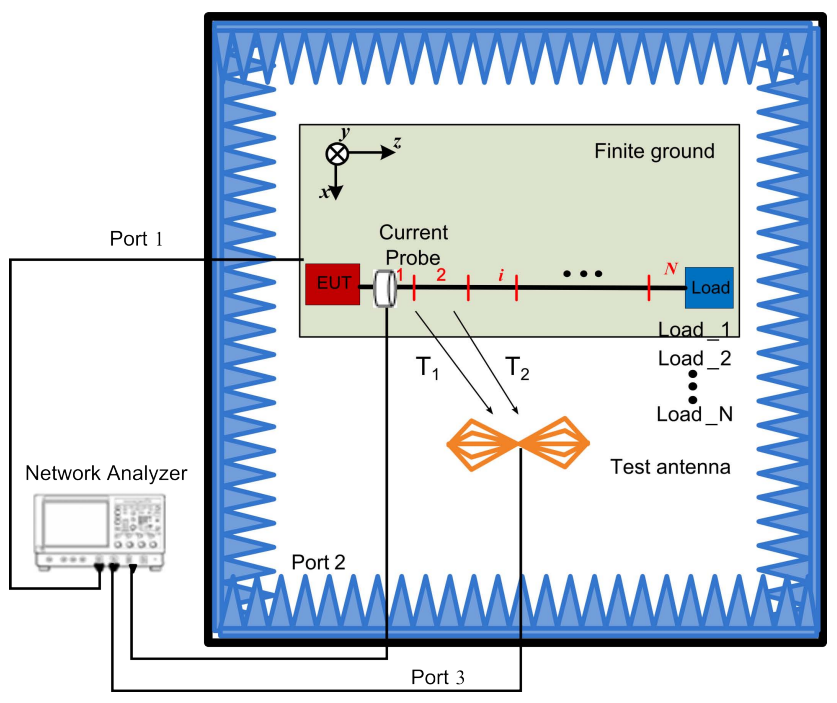

Fig. 4. Transfer factor measurement setup. respectively planar structure field scan methods are applicable (Rinas and Frei, 2012; Chen et al., 2009; Thomas et al., 2012). Here the current distribution on the PCB can be found through measuring the spatial magnetic field above the PCB. Including geometry properties and knowledge of possible current paths the radiation model can be optimized.

As the cable and PCB models from near-field scan methods allow emission calculation in free space but the standardized ALSE antenna measurements are done in chamber measurement environment with influencing factors listed above, the alternative methods must consider in the first step the chamber effects in order to produce comparable results (Smith and Frazier, 1998). Influence factors are interaction with antenna (A), reflections from the walls of anechoic chamber (B), currents in table edges (C) and emission from measurement equipment cables (D), as shown in Fig. 1. Methods to determine correction and transfer functions based on measurement data are presented in this paper. 

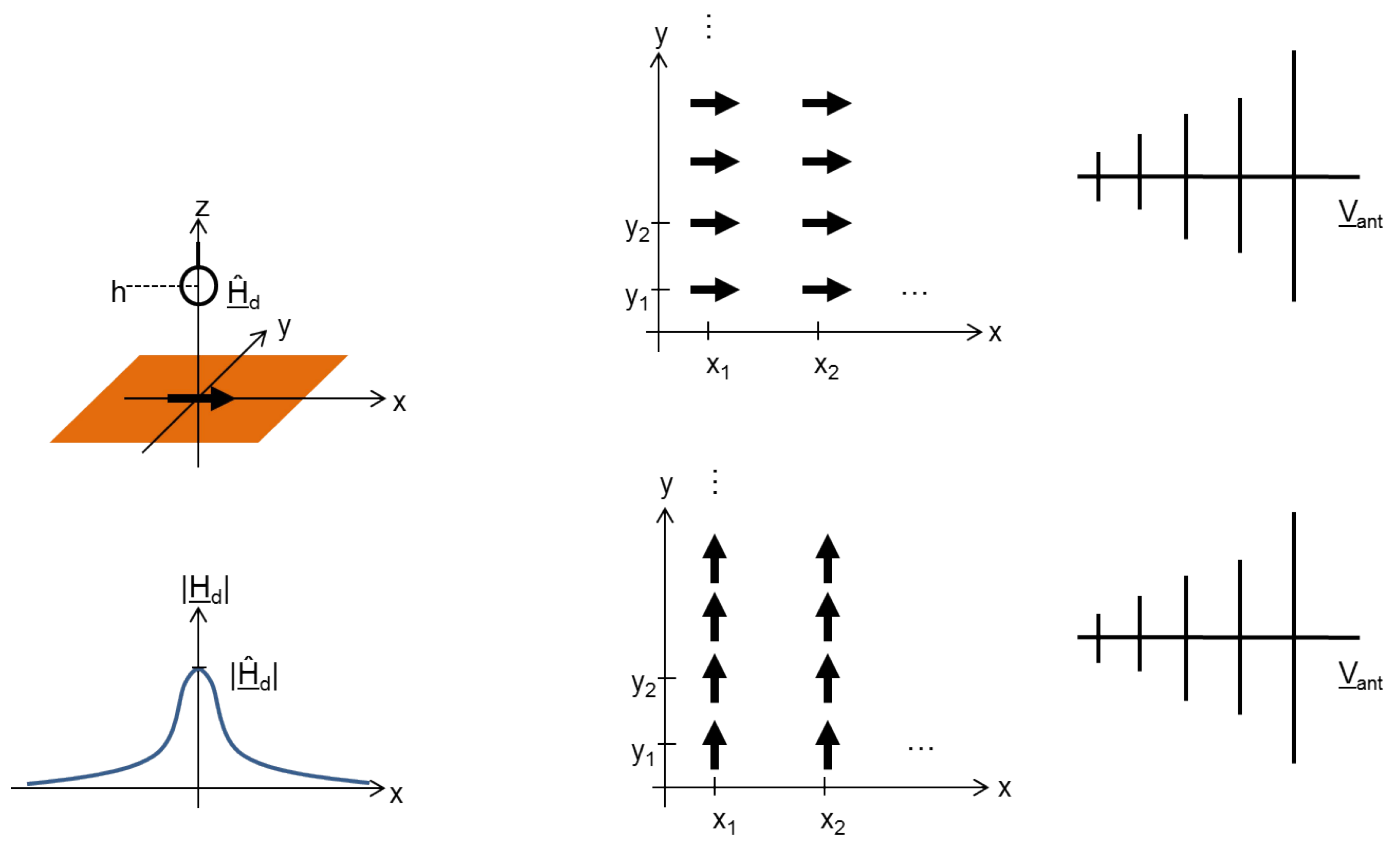

Fig. 5. Hertzian dipole configurations for calibration process. (a) Hertzian dipole and dipole near field. (b) Calibration in $x$ - and $y$-direction for transfer function computation.

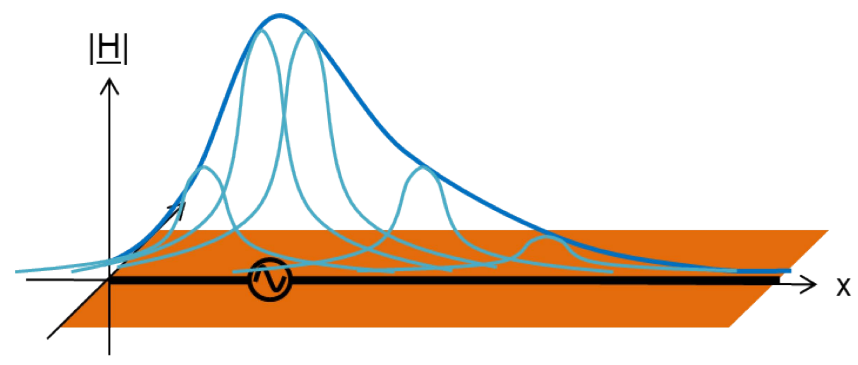

Fig. 6. Magnetic near field in each row and column of PCB can be approximated by varying position of a set of dipoles.

\section{Methods for substituting of antenna emission measurement}

In the following sections the basic methods for antenna emission measurement substitution are introduced. The differences between cable bundle and PCB measurements are discussed.

\subsection{Cable bundles}

In a radiation model a cable bundle can be divided into a set of short dipoles (Jia et al., 2012) as shown in Fig. 2. In this model, the influence of ground is incorporated into the radiation model using mirror theory which is actually different from real test configuration with more complex environment, i.e. metallic table of limited size, large test antenna, non-perfect absorbing materials, and so on. Aiming at inte- grating real influence factors in radiation models, we developed two methods to correct calculated field result.

The proposed method calculates the absolute electric field at a reference point, the antenna center point. However, the test antenna converts the electric field around the antenna to a receiving voltage. In order to get the antenna field, the measured voltage should be multiplied with the antenna factor (AF) provided by the antenna manufacturer. In real test configurations, the antenna is located in the near field from the cable bundle in a wide frequency range, while AF from manufacture is calibrated in far field in an open area test site. Therefore this antenna factor will not be accurate when used in near field test. We first measure antenna receiving voltage radiated from cable bundle in standard configuration as shown in Fig. 3a and then use AF from manufacturer to obtain electric field at reference point ( $1 \mathrm{~m}$ distant to cable bundle). Subsequently we simulate the radiation from a verified MoM cable model to acquire electric field at the same point, as shown in Fig. 3b. Finally we can calculate the correction factor according to Eq. (1):

$$
\begin{aligned}
& E_{\text {measure }}=V_{\text {measure }}+A F(\mathrm{~dB}) \\
& K=E_{\text {measure }}-E_{\text {simulation }}(\mathrm{dB})
\end{aligned}
$$

Another method is based on a set of measured transfer functions from known currents and measured antenna voltages, as shown in Fig. 4. We scan the currents at $N$ different nodes along the cable bundle when the cable is terminated with $N$ different loads. $N \times N$ currents and $N$ antenna voltages can be acquired, and then we can solve an equation system (4) to get a set of transfer functions $\left[T_{1}, T_{2}, \ldots T_{i}, \ldots, T_{N}\right]$. In 


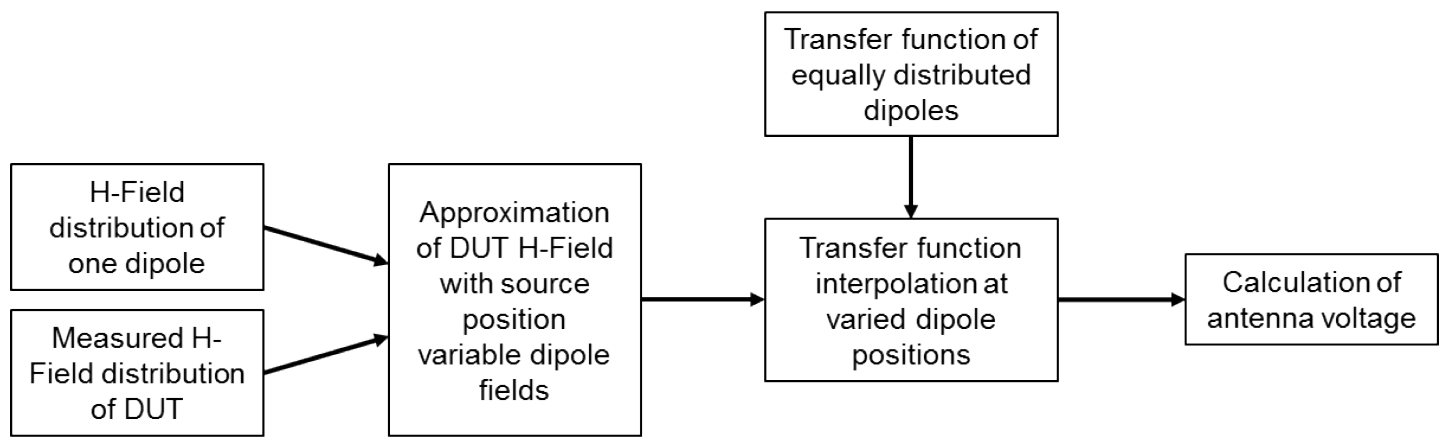

Fig. 7. Process diagram of antenna emission measurement substitution.

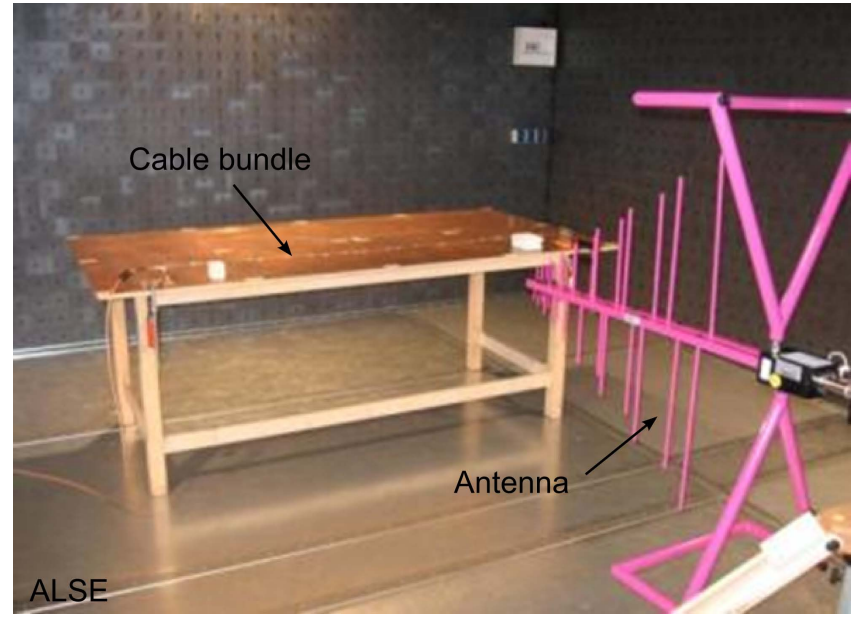

Fig. 8. Setup for measurement of transfer functions and verification of the method.

real test, we can directly evaluate the antenna voltage based on scanned currents of cable bundle (amplitude and phase) and transfer functions $T_{i}$.

$S_{21}=2 \times \frac{V_{2}}{V_{S}}=2 \times \frac{I_{i} \times Z t}{V_{S}}$

$S_{31}=2 \times \frac{V_{3}}{V_{S}}=2 \times \frac{U_{i}}{V_{S}}(i=1 \cdots N)$

$\left[\begin{array}{cccc}I_{\text {Load_1 }}^{1} & I_{\text {Load_1 }}^{2} & \cdots & I_{\text {Load_1 }}^{N} \\ I_{\text {Load_2 }}^{1} & I_{\text {Load_2 }}^{2} & \cdots & I_{\text {Load_2 }}^{N} \\ \vdots & \vdots & \vdots & \vdots \\ I_{\text {Load_N }}^{1} & I_{\text {Load_N }}^{2} & \cdots & I_{\text {Load_N }}^{3}\end{array}\right]\left[\begin{array}{c}T_{1} \\ T_{2} \\ \vdots \\ T_{N}\end{array}\right]=\left[\begin{array}{c}U_{1} \\ U_{2} \\ \vdots \\ U_{N}\end{array}\right]$

\subsection{PCB}

In Sect. 2.1 it is shown that the calibration between cable current and test antenna voltage can be computed by dividing a cable in $N$ segments and determining a transfer function for

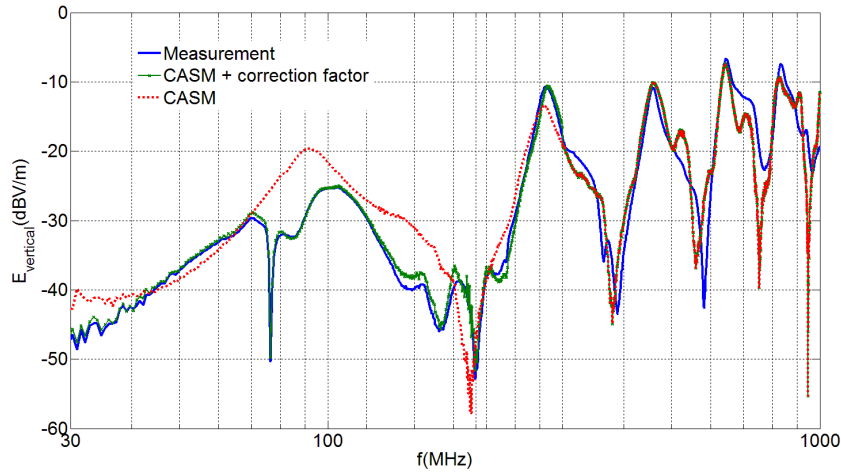

Fig. 9. Electric field in vertical direction of measurement and CASM.

each segment. In the same way a PCB can be discretised in $N \times M$ squares and transfer functions can be computed for each square in $x$ - and $y$-current direction, as shown in Fig. 5b.

As reference for calibration process and theoretical considerations a dipole antenna is applied. The magnetic near field in a plane above the dipole can be calculated or measured (Rinas et al., 2011), as shown in Fig. 5a. The transfer function for each square is calculated by varying the position of the reference dipole along the squares, as shown in Fig. 5b.

$T_{n m, x}=\frac{V_{\text {ant }}}{H_{n m, x}}$

$T_{n m, y}=\frac{V_{\text {ant }}}{H_{n m, y}}$

where $T_{n m, x}$ and $T_{n m, y}$ are the transfer functions for the squares in $x$ - and $y$-direction, $V_{\text {ant }}$ is the corresponding antenna voltage and $H_{n m, x}$ and $H_{n m, y}$ the measured magnetic fields.

To get the magnetic near field of the PCB it is scanned with near field scanner in $x$ - and $y$-direction. The resolution of near field scanning is $K \times L$ with $K \gg N$ and $L \gg M$. With the use of an optimization algorithm a distribution of 

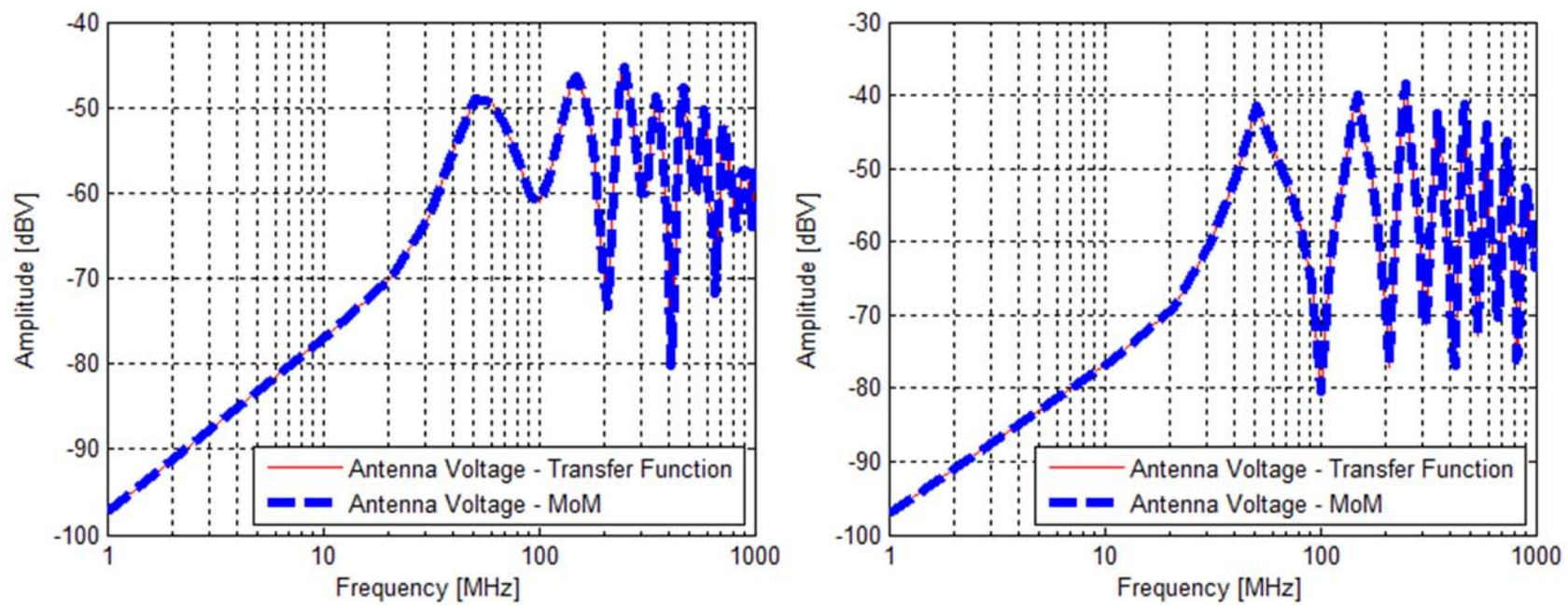

Fig. 10. Antenna voltage from MoM and transfer function method. (a) Load: $1600 \Omega$. (b) Load: $400 \Omega-500 \mu \mathrm{H}-200 \mathrm{pF}$.

a dipoles can be found which best fits the magnetic field of each scanned row and column, as shown in Fig. 6. After that the transfer function for each dipole at new positions is interpolated. It should be noted that approximating the magnetic field in amplitude and phase close to the source structure is equal to approximating the current distribution. Knowing current distribution voltage distribution is known to and magnetic and electric field can be calculated. Superposition of transfer function calibrated squares leads to calculation of resulting antenna voltage.

$V_{\mathrm{ant}}=\sum T_{n m} H_{n m}$

The process diagram for PCBs is shown in Fig. 7.

\section{Results}

To show the applicability of the presented methods parts of results are based on Method of Moments (MoM) simulation data.

\subsection{Cable bundles}

In order to verify the proposed methods of correction factor and transfer function, we use an EMC antenna to test radiation from a simple cable, as shown in Fig. 8. The CASM method (Jia et al., 2012) was used to acquire amplitude and phase of currents along the cable and then electric field was calculated at the reference point using dipole model. We compared the results from measurement (measured antenna voltage plus antenna factor), CASM with mirror theory and CASM based on correction factor, as shown in Fig. 9. It can be seen that the resonances around $70 \mathrm{MHz}$ and $350 \mathrm{MHz}$ originating from test environment can be considered through correction factor. The deviation in high frequency is mainly

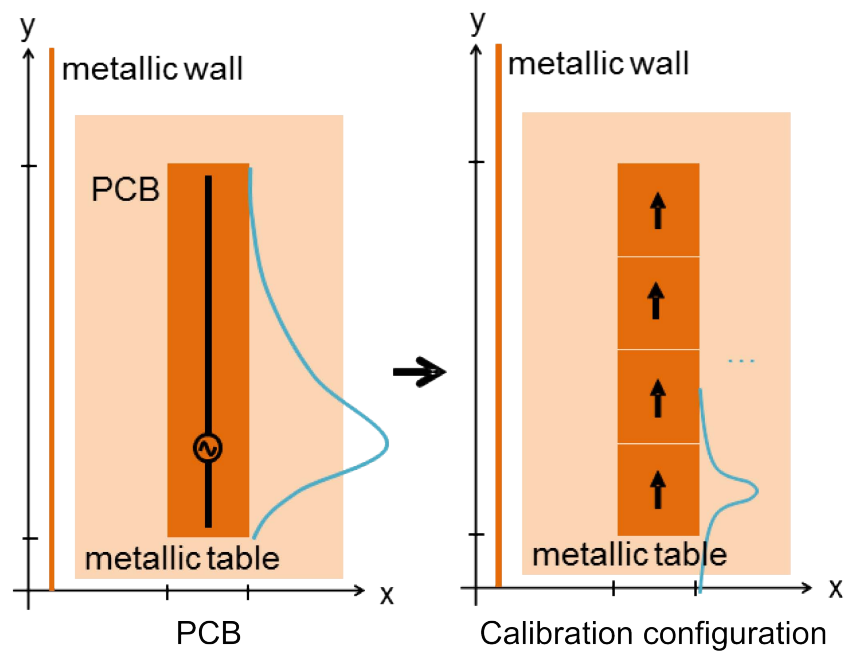

Fig. 11. Transfer function calibration of PCB with set of dipoles.

from measured current amplitude error and CASM algorithm itself.

For transfer function method, we use here simulation results by MoM method. The cable has a length of $1.5 \mathrm{~m}$, a height to metallic table of $0.05 \mathrm{~m}$, and is terminated by 17 different loads: short, open, $50 \Omega, 100 \times M \Omega(M=$ $[1,2, \ldots, 14])$. We simulate the currents at different nodes of cable terminated by 17 different loads; also a MoM model of a biconical antenna was created to simulate antenna voltages. According to Eq. (4) the transfer function $T_{N}$ can be determined. In order to verify the transfer functions, we simulated two cases: the cable is terminated by $1600 \Omega$ or a series impedance of $400 \Omega-500 \mathrm{nH}-200 \mathrm{pF}$. Figure 10 presents antenna voltage measured directly and antenna voltage computed using the transfer function. It can be seen, the results from transfer function method agree well with direct antenna 


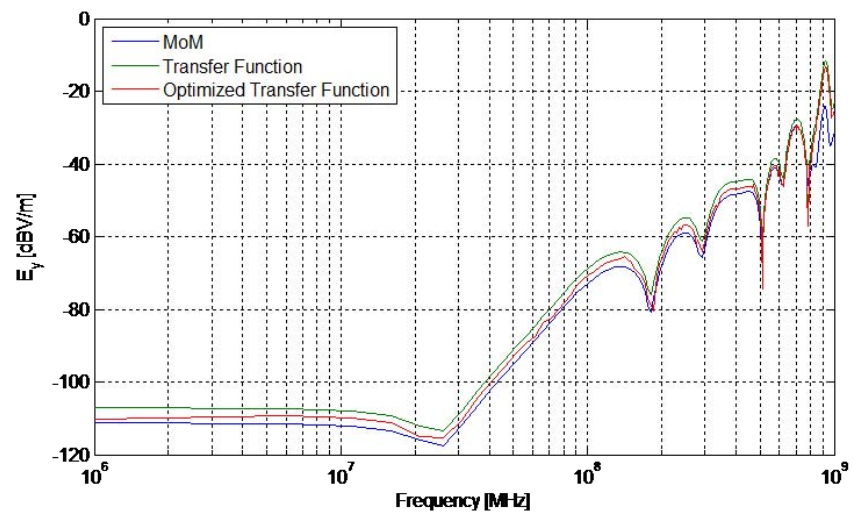

Fig. 12. Electric field calculated with transfer function and optimized transfer function in comparison to electric field from MoM.

voltage measurements. But it is noted that the scanned current and voltage antenna here are based on simulation results, which are free from noise, which can not be avoided in real measurements. The current and antenna voltage are both complex numbers in Eq. (4) which need amplitude and phase simultaneously.

\subsection{PCB}

For transfer function substitution, we present simulation results by MoM method. The test PCB consists of a single cable with a length of $0.16 \mathrm{~m}$, a height over metallic plane of $5 \mathrm{~mm}$, and two open ends. It is fed at length of $0.04 \mathrm{~m}$ with a supply voltage of $1 \mathrm{~V}$. Transfer function calibration is done with four dipoles of finite length in a height of $5 \mathrm{~mm}$ above metallic plane, as shown in Fig. 11. According to Eqs. (5)(7) transfer functions $T_{n m}$ are computed and antenna voltage is calculated. To get better results approximation to near field distribution and transfer function interpolation is done.

Figure 12 presents the results of the substitution process. It can be seen that the electric field computed with transfer function shows good results up to a frequency of $800 \mathrm{MHz}$. Using near field approximation and transfer function interpolation the results can be improved in the entire frequency range.

\section{Conclusions}

In this paper methods for substituting antenna emission measurements with field and cable scan methods are introduced. Influencing factors like interaction with antenna, wall reflections, table currents and emission from measuring cable can be considered by use of measurement data from "calibration structures" and transfer functions. Cable bundles and PCBs are divided in segments and the transfer function can be computed for each of these segments. Superposition of the emissions gives the desired antenna voltage.

First results, partly based on MoM simulation data, are shown which proves the applicability of the proposed method. The investigations have to be extended in future.

Acknowledgements. The reported $\mathrm{R}+\mathrm{D}$ work was carried out within the CATRENE project CA310 EM4EM (Electromagnetic Reliability and Electronic Systems for Electro Mobility). This particular research is supported by the BMBF (Bundesministerium fuer Bildung und Forschung) of the Federal Republic of Germany under grant 16 M3092 I. The responsibility for this publication is held by the authors only.

\section{References}

Chen, Q., Chakarothai, J., and Sawaya, K.: Estimation of Current Distribution by Near-Field Measurement, CEEM, China, 2009.

CISPR 25 Ed. 3: Vehicles, boats and internal combustion engines Radio disturbance characteristics - Limits and methods of measurement for the protection of on-board receivers, IEC, 2007.

Jia, J., Rinas, D., and Frei, S.: Prediction of Radiated Fields from Cable Bundles based on Current Distribution Measurements, EMC Europe, Rome, 2012.

Smith, W. T. and Frazier, R. K.: Prediction of Anechoic Chamber Radiated Emissions Measurements Through Use of EmpiricallyDerived Transfer Functions and Laboratory Common-Mode Current Measurements, EMC, Denver, 1998.

Thomas, D. W. P., Obiekezie, C., Greedy, S., Nothofer, A., and Sewell, P.: Characterisation of Noisy Electromagnetic Fields from Circuits using the Correlation of Equivalent Sources, EMC Europe, Rome, 2012.

Rinas, D. and Frei, S.: Methoden zur Optimierung von Störaussendungsmodellen für Platinenstrukturen auf Basis von Nahfeldmessdaten, EMV Düsseldorf, Germany, 2012.

Rinas, D., Niedzwiedz, S., Jia, J., and Frei, S.: Optimization Methods for Equivalent Source Identification and Electromagnetic Model Creation based on Near-Field Measurements, EMC Europe, York, 2011. 